\title{
SMALL ISOTOPIES IN EUCLIDEAN SPACES AND 3-MANIFOLDS ${ }^{1}$
}

\author{
BY JAMES KISTER
}

\author{
Communicated by Edwin Moise, October 6, 1959
}

1. Introduction. The general type of question considered here is: what homeomorphisms of a space or of a set are obtained by isotopic deformations of a space by a small amount. Although questions of this type have only recently been treated explicitly and for their own sake (e.g. $[2 ; 3 ; 5 ; 6 ; 7])$ they had been handled implicitly in work done by Alexander [1] and Kneser [4] some 35 years ago. In fact this paper owes much to the method of Alexander, rediscovered in a slightly different form.

2. Definitions. Let $M$ be a manifold with boundary having a metric $d .{ }^{2}$ Denote by $\mathfrak{H C}(M)$ the set of all homeomorphisms of $M$ onto itself. Define a function $\rho$ of $\mathfrak{F} \times \mathfrak{F C}$ into the extended real number system as follows: $\rho(f, g)=\sup _{x \in M} d(f(x), g(x)) . f$ and $g$ are $\epsilon$-isotopic if there is an isotopy $H_{t}, t \in I$, so that $H_{0}=f, H_{1}=g$ and if $t_{1}, t_{2} \in I$ then $\rho\left(H_{t_{1}}, H_{t_{2}}\right) \leqq \epsilon$.

\section{Results.}

THEOREM 1. If $f$ and $g$ are in $\mathfrak{H}\left(E^{n}\right)$ and $\rho(f, g)=\epsilon<\infty$ then $f$ and $g$ are $\epsilon$-isotopic.

Proof. By the right invariance of $\rho$ it follows that $\rho(f, g)=\rho\left(1, g f^{-1}\right)$ and if 1 and $g f^{-1}$ are $\epsilon$-isotopic under $H_{t}$ then $f$ and $g$ are $\epsilon$-isotopic under $H_{t} f$. Hence it suffices to prove the theorem for $f=1$. In this case, using vector notation for points in $E^{n}$, let $H_{t}(x)=t g(x / t)$ for $0<t \leqq 1$ and let $H_{0}=1$. The continuity of $H_{t}(x)$ in $t$ and $x$ is clear for $t>0$ and assured for $t=0$ by $d\left(x, H_{t}(x)\right)=t d(x / t, g(x / t)) \leqq t \epsilon$.

This generalizes to $E^{n}$ (and slightly strengthens) a recent result of Sanderson for $E^{3}$ [7]. Alexander's result follows immediately by restricting the isotopy $H_{t}$ to the unit ball in $E^{n}$.

Another direction generalization can take is:

\section{THEOREM 2. Let $M$ be an arbitrary 3-manifold with boundary having a}

1 The author is indebted to Professor R. H. Bing for suggesting the problem answered by Theorem 1 for $n=3$, and for directing the thesis, partially summarized here, to which the solution of that problem inexorably led. This research was supported by a Gulf Research and Development Company Fellowship.

${ }^{2}$ All Euclidean spaces $E^{n}$ will be assumed to have the usual metric. For more general manifolds the metric will be specified as needed. 
triangulation $\Sigma$. Let $d b$ the barycentric metric determined by $\Sigma$ and let $\rho$ be as defined above. Given any $\epsilon>0$ there is $a \delta>0$ so that if $f, g \in \mathcal{H C}(M)$ and $\rho(f, g)<\delta$, then $f$ and $g$ are $\epsilon$-isotopic.

Proof. Only a sketch will be given here since the proof is quite long.

Again it suffices to let $f=1$. The proof is in four stages. If we restrict $g$ to be close to 1 then on each 3 -simplex $T$ in $\Sigma$ we can replace $g$ by $g^{\prime}$ where $g^{\prime} \mid \mathrm{Bd} T=1$ and $g^{\prime}$ agrees with $g$ except in a small neighborhood of Bd $T$. An Alexander-type isotopy on each $T$ takes $g^{\prime}$ onto 1 moving no point far. The global isotopy has the effect of deforming $g$ to a homeomorphism $g_{1}$ which is 1 except in a small neighborhood of the 2-skeleton. Next using [6] $g_{1}$ is modified to get $g_{1}^{\prime}$ which is the same as $g_{1}$ on cubes built over the 2 -simplexes in $\Sigma$ and is different from $g_{1}$ only near the 1-skeleton of $\Sigma$. An isotopy is pieced together again which deforms $g_{1}$ to a homeomorphism $g_{2}$ which is 1 except in a small neighborhood of the 1-skeleton. Two more reductions, near the 1skeleton and 0 -skeleton respectively, which are described on disjoint cubes near the 1-simplexes and vertices respectively, take $g_{2}$ onto the identity.

COROLlARy 1. If $M$ is a compact 3-manifold with boundary, then $h$ is isotopic to 1 if and only if $h=h_{k} h_{k-1} \cdots h_{2} h_{1}$ where each $h_{i}$ is the identity outside a polyhedral 3-cell.

CoRollary 2. If $L$ is a tame compact 2-manifold in any 3-manifold $M$ and $\epsilon>0$, there is $a \delta>0$ so that if $h$ is any homeomorphism of $L$ into $M$ moving no point more than $\delta$ and if $h(L)$ is tame, then there is an $\epsilon$-isotopy of $M$ taking $h(L)$ onto $L$ pointwise and moving no point outside an $\epsilon$-neighborhood of $L$.

This makes use of and generalizes a result of Sanderson [6].

CoRollary 3. If $M$ is a 3-manifold having triangulation $\Sigma$ and $\epsilon>0$ there is a $\delta>0$ so that if $h$ is a homeomorphism of the 2-skeleton $K$ of $\Sigma$ into $M$ moving no point more than $\delta$ and such that $h(K)$ is tame, then there is an $\epsilon$-isotopy of $M$ taking $h(K)$ onto $K$ pointwise.

Question. In Corollary 3 can $K$ be replaced by a 2-complex having no local separating points?

The author has been informed that G. M. Fisher and M. E. Hamstrom separately have obtained Theorem 2 for $M$ a compact 3-manifold with boundary and that the former also obtained Corollary 1. 


\section{REFERENCES}

1. J. W. Alexander, On the deformation of an n-cell, Proc. Nat. Acad. Sci. vol. 9 (1923) pp. 406-407.

2. E. Dyer and M. E. Hamstrom, Regular mappings and the space of homeomorphisms on a 2-manifold, Duke Math. J. vol. 25 (1958) pp. 521-531.

3. M. K. Fort, $A$ proof that the group of all homeomorphisms of the plane onto itself is locally-arcwise connected, Proc. Amer. Math. Soc. vol. 1 (1950) pp. 59-62.

4. H. Kneser, Die Deformationssätze der einfach zusammenhängenden Flöchen, Math. Z. vol. 25 (1926) pp. 362-372.

5. J. H. Roberts, Local arcwise connectivity in the space $H^{n}$ of homeomorphisms of $S^{n}$ onto itself, Summary of Lectures, Summer Institute on Set Theoretic Topology, Madison, Wisconsin, 1955, p. 100.

6. D. E. Sanderson, Isotopy in 3-manifolds. II. Fitting homeomorphisms by isotopy, Duke Math. J. vol. 26 (1959) pp. 387-396.

7. - Isotopy in 3-manifolds. III. Connectivity of spaces of homeomorphisms, to appear in Proc. Amer. Math. Soc.

UNIVERSITY OF WISCONSIN AND

UNIVERSITY OF MICHIGAN 